\title{
BETULA: Numerically Stable CF-Trees for BIRCH Clustering ${ }^{\star}$
}

\author{
Andreas Lang ${ }^{[0000-0003-3212-5548]}$ and Erich Schubert ${ }^{[0000-0001-9143-4880]}$ \\ TU Dortmund University, Dortmund, Germany \\ \{andreas.lang, erich.schubert\}@tu-dortmund.de
}

\begin{abstract}
BIRCH clustering is a widely known approach for clustering, that has influenced much subsequent research and commercial products. The key contribution of BIRCH is the Clustering Feature tree (CF-Tree), which is a compressed representation of the input data. As new data arrives, the tree is eventually rebuilt to increase the compression. Afterward, the leaves of the tree are used for clustering. Because of the data compression, this method is very scalable. The idea has been adopted for example for $k$-means, data stream, and density-based clustering. Clustering features used by BIRCH are simple summary statistics that can easily be updated with new data: the number of points, the linear sums, and the sum of squared values. Unfortunately, how the sum of squares is then used in BIRCH is prone to catastrophic cancellation.

We introduce a replacement cluster feature that does not have this numeric problem, that is not much more expensive to maintain, and which makes many computations simpler and hence more efficient. These cluster features can also easily be used in other work derived from BIRCH, such as algorithms for streaming data. In the experiments, we demonstrate the numerical problem and compare the performance of the original algorithm compared to the improved cluster features.
\end{abstract}

\section{Introduction}

The BIRCH algorithm $[23,24,22]$ is a widely known cluster analysis approach, that won the 2006 SIGMOD Test of Time Award. It scales well to big data even with limited resources because it processes the data as a stream and aggregates it into a compact summary of the data. BIRCH has inspired many subsequent works, such as two-step clustering [10], data bubbles [7], and stream clustering methods such as CluStream [1] and DenStream [9]. Clustering is the unsupervised learning task aimed at discovering potential structure in a data set when no labeled data or pattern examples are available. It is inherently underspecified and subjective $[12,5]$ and, unfortunately, also very difficult to evaluate. Instead, it is best approached as explorative data analysis, generating hypotheses about potential structures in the data, that afterward need to be verified by some

\footnotetext{
* Part of the work on this paper has been supported by Deutsche Forschungsgemeinschaft (DFG) within the Collaborative Research Center SFB 876 "Providing Information by Resource-Constrained Analysis", project A2
} 
other procedure, which is domain-specific and may require a domain expert to inspect the results. Many clustering algorithms and evaluation measures have been proposed with unclear advantages of one over another. Because many of the underlying problems (e.g., $k$-means clustering) are NP-hard, we often use approximation techniques and great concern is directed at the scalability.

Scalability is where the BIRCH algorithm shines. It is a multi-step procedure for numerical data that first aggregates the data into a tree-based data structure much smaller than the original data. This condensed representation is then fed into a clustering method, which now is faster because of the reduced size. The main contribution of BIRCH is a flexible logic for aggregating the data so that an informative representation is retained even when the size is reduced substantially.

When studying BIRCH closely, we noticed that it is susceptible to a numerical problem known as "catastrophic cancellation". This arises when two large and similar floating-point values are subtracted: many bits of the significand cancel out, and only few bits of valid result remain. In this paper, we show how to avoid this numerical problem and demonstrate that it can arise in real data even at low dimensionality. We propose a replacement cluster feature tree (BETULA) that does not suffer from this numeric problem while retaining all functionality. Furthermore, it is often even easier to use. This structure can easily be integrated into most (if not all) derived methods, in particular also for data streams.

\section{Related Work}

The BIRCH algorithm was presented at the SIGMOD conference [23], then expanded in a journal version [24]. Still, both versions omit integral details of the algorithm (e.g., Eqs. 15 to 17 below to compute distances using cluster features), which are found only in their technical report [22] or their source code. Nevertheless, the intriguing ideas of the clustering features and the CF-Tree inspired a plethora of subsequent work. Bradley et al. [6] use the same "clustering features" as BIRCH, but call them "sub-cluster sufficient statistics". The CF-Tree has also been used for kernel density estimation [25], with a threshold set on the variance to guarantee approximation quality. In two-step clustering [10], BIRCH is extended to mixed data, by adding histograms over the categorical variables.

Because BIRCH is sequentially inserting data points into the CF-tree, the tree construction can be suspended at any time. The leaves can then be processed with a clustering algorithm; when new data arrives the tree construction is continued and we trivially obtain a stream clustering algorithm [15]. CluStream [1] extends this idea with pyramidal time frames to enable the clustering of parts of the data stream by integrating temporal information. HPStream [2] extends CluStream to projected/subspace clustering. DenStream [9] uses clustering features for density-based stream clustering to detect clusters of arbitrary shape (in contrast to earlier methods that focus on $k$-means-style clustering). Breunig et al. [8] adopt clustering features to perform hierarchical density-based OPTICS clustering [3] on large data. The ClusTree [17] combines R-trees with BIRCH clustering features to process data streams. BICO [13] aims at improv- 
ing the theoretical foundations (and hence, performance guarantees) of $\mathrm{BIRCH}$ by combining it with the concept of coresets. For this, it is necessary to add reference points to the clustering features and use a strict radius threshold.

\section{BIRCH and BETULA}

In this section, we will describe the basic BIRCH tree building algorithm, and introduce the changes made for BETULA to become numerically more reliable.

\subsection{BIRCH Clustering Features}

The central concept of BIRCH is a summary data structure known as Clustering Features $\mathrm{CF}^{\mathrm{BIRCH}}=(\boldsymbol{L} \boldsymbol{S}, S S, N)$. Each clustering feature represents $N$ data points, summarized using the linear sum vector $\boldsymbol{L} \boldsymbol{S} \in \mathbb{R}^{d}$ (with $L S_{i}=\sum_{x} x_{i}$ ), the sum of squares $S S \in \mathbb{R}$ (originally not a vector, but a scalar $S S=\sum_{i} \sum_{x} x_{i}^{2}$ ) and the count $N \in \mathbb{N}$. The center of a clustering feature can be trivially computed as $L S / N$. By the algebraic identity $\operatorname{Var}(X)=E\left[X^{2}\right]-E[X]^{2}$, BIRCH computes the variance of a clustering feature as $\operatorname{Var}(X)=\frac{1}{N} S S-\left(\frac{1}{N} \sum_{i} L S_{i}\right)^{2}$. We will discuss the numerical problems with this approach in Section 3.5.

A new data sample $x$ can be easily integrated into the clustering feature using $\mathrm{CF}^{\mathrm{BIRCH}}+\boldsymbol{x}=\left(\boldsymbol{L} \boldsymbol{S}+\boldsymbol{x}, S S+\sum_{i} x_{i}^{2}, N+1\right)$. Because all of these are sums, two clustering features can also easily be combined (c.f., additivity theorem in [23]) $\mathrm{CF}_{A}^{\mathrm{BIRCH}}+\mathrm{CF}_{B}^{\mathrm{BIRCH}}=\left(\boldsymbol{L} \boldsymbol{S}_{A}+\boldsymbol{L} \boldsymbol{S}_{B}, S S_{A}+S S_{B}, N_{A}+N_{B}\right)$. A single data point $\boldsymbol{x}$ can hence be interpreted as the clustering feature containing $\left(\boldsymbol{x}, \sum_{i} x_{i}^{2}, 1\right)$.

\subsection{Clustering Feature Tree (CF-Tree)}

The cluster features are organized in a depth-balanced tree called CF-Tree. A leaf stores a set of clustering features (each representing one or many data points), while the inner nodes store the aggregated clustering features of each of its children. The tree is built by sequential insertion of data points (or, at a rebuild, the insertion of earlier clustering features). The insertion leaf is found by choosing the "nearest" clustering feature at each level (five different definitions of closeness will be discussed in Section 3.4). Within the leaf node, the data point is added to the best clustering feature if it is within the merging "threshold", otherwise a new clustering feature is added to the leaf. Leaves that exceed a maximum capacity are split, which can propagate to higher levels of the tree and cause the tree to grow when the root node overflows. If the tree exceeds the memory limit, a new tree is built with an increased merging threshold by reinserting the existing clustering features of the leaf level. After modifying a node, the aggregated clustering features along the path to the root are updated.

The discussion of BIRCH in textbooks ends with the CF-Tree, although we do not yet have clusters. This is because the outstanding idea of BIRCH is that of data aggregation into clustering features, and we can run different clustering 
algorithms afterward. The BIRCH authors mention hierarchical clustering, $k$ means, and CLARANS [19]. For best results, we would want to use an algorithm that not only uses the mean of the clustering feature, but that also uses the weight and variance. The weight can be fairly easily used in many algorithms, but the variance is less obvious to integrate. In Section 3.7 we will propose how to perform Gaussian Mixture Modeling and use the variance information.

\subsection{BETULA Cluster Features}

The way variance is computed from BIRCH cluster features using the popular equation $\operatorname{Var}(X)=E\left[X^{2}\right]-E[X]^{2}$ is prone to the numerical problem known as "catastrophic cancellation". This equation can return zero for non-constant data, and because of rounding even negative values (and hence, undefined standard deviation). In the context of BIRCH, we cannot resort to the numerically more reliable textbook definition for variance, $\operatorname{Var}(X):=\frac{1}{N} \sum(x-\mu)^{2}$, because this requires two passes over the data set (one to find $\mu$, then one for Var). But we also cannot just ignore the problem, because not all clustering features will be close to 0, where the numerical accuracy is not a problem. Schubert and Gertz [20] discuss methods to compute variance and covariance for weighted data, which forms the base for our approach. For this, they collect three running statistics, very similar to the three components of BIRCH clustering features: (i) the sum of weights, (ii) the weighted mean (centroid vector), and (iii) the weighted sum of squared deviations from the mean. Clearly (i) corresponds to $N$ in the clustering feature, (ii) is equivalent to $L S / N$, but (iii) is $S:=\sum_{x} n_{x}\|x-\mu\|^{2}$ (where $n_{x}$ is the weight of the data point, often simply 1 ). Hence, we propose the following replacement cluster feature for BETULA:

$$
\mathrm{CF}^{\mathrm{BETULA}}:=(n, \mu, S)
$$

where $n$ is the aggregated weight of all data points (BETULA also allows for weighted data samples), $\mu$ denotes the current mean vector, and $S$ is the sum of squared deviations from the mean. The last component can either be a scalar value as in BIRCH (the sum over all components) or a vector of squared deviations. For our experiments, we chose the latter option; a similar modification to BIRCH can be found in various publications (e.g., $[1,2,9,17,16])$. A single data point of weight $n_{x}$ is equivalent to a cluster feature $\mathrm{CF}_{x}^{\mathrm{BETLA}}=\left(n_{x}, x, 0\right)$ (because it has zero deviation from the mean). Similar to the additivity theorem of BIRCH, we can efficiently combine two BETULA cluster features into one:

$$
\begin{aligned}
& n_{A B}=n_{A}+n_{B} \\
& \mu_{A B}=\mu_{A}+\frac{n_{B}}{n_{A B}}\left(\mu_{B}-\mu_{A}\right) \\
& S_{A B}=S_{A}+S_{B}+n_{B}\left(\mu_{A}-\mu_{B}\right)\left(\mu_{A B}-\mu_{B}\right)
\end{aligned}
$$

The derivation of these equations follows directly from the update equations for the weighted (co-) variance of [20]. Because their experiments indicate that using the sum of squared deviations, $S:=\sum_{x} n_{x}(x-\mu)^{2}$ has slight computational advantages, we follow suit. We could also have stored $\operatorname{Var}=S / n$ instead (we did not measure a noticeable performance difference between these two options). 


\subsection{Distance and Absorption Measures}

The BIRCH algorithm uses two different measures during tree construction. The first is a distance between two clustering features, which is used to find the closest leaf in the tree. The second is an absorption criterion, used together with a threshold to decide when to add the new data to an existing or as a new clustering feature. Both measures can be defined on the original data, but also in terms of the clustering feature values to compute them efficiently.

Distance Measures: BIRCH proposes five different distance measures enumerated as D0 to D4. The first two correspond simply to the Euclidean distance of the centers (D0) and the Manhattan distance of the centers (D1). The third, average inter-cluster distance (D2), is based on the quadratic mean distance between points of different clusters, while the average intra-cluster distance (D3) uses the quadratic mean distance within the combined cluster. Variance-increase distance (D4) is the variance of the resulting cluster minus the variance of the separated clusters. Similar ideas can be found in hierarchical clustering: centroid linkage (D0, D1), average linkage (D2, D3), and Ward linkage (D4).

$$
\begin{aligned}
& \operatorname{D} 0(A, B)=\left\|\mu_{A}-\mu_{B}\right\|=\sqrt{\sum_{i}\left(\mu_{A, i}-\mu_{B, i}\right)^{2}} \\
& \operatorname{D1}(A, B)=\left\|\mu_{A}-\mu_{B}\right\|_{1}=\sum_{i}\left|\mu_{A, i}-\mu_{B, i}\right| \\
& \operatorname{D} 2(A, B)=\sqrt{\frac{1}{n_{A} n_{B}} \sum_{x \in A} \sum_{y \in B}\|x-y\|^{2}} \\
& \operatorname{D} 3(A, B)=\sqrt{\frac{1}{n_{A B}\left(n_{A B}-1\right)} \sum_{x, y \in A B}\|x-y\|^{2}} \\
& \operatorname{D} 4(A, B)=\sqrt{\sum_{x \in A B}\left\|x-\mu_{A B}\right\|^{2}-\sum_{x \in A}\left\|x-\mu_{A}\right\|^{2}-\sum_{x \in B}\left\|x-\mu_{B}\right\|^{2}}
\end{aligned}
$$

Absorption Criteria: Absorption in BIRCH is based on a second criterion and a threshold. Conceptually, the threshold can be seen as a maximum radius of a cluster feature; if adding a point would increase the radius beyond the allowed maximum, a new cluster feature is created instead of merging. Intuitively, the radius should be defined as $\max _{x}\|x-\mu\|$; but this value cannot be efficiently computed from the summary statistics. Instead, the "radius" can be approximated using different criteria. In BIRCH, these criteria were defined on a single clustering feature $A B$; they are computed by virtually merging two clustering features and evaluating the criteria on the result. We can easily remove the distinction between distance and absorption criteria, but one may nevertheless want to choose them differently (e.g., choosing the nearest leaf by Euclidean distance, but thresholding on minimum variance), as they serve a different purpose. The first criterion proposed in BIRCH is called "radius" R (Eq. 10), the second is the "diameter" D (Eq. 11). Both this "radius" and "diameter" are not maximum values, but averages: the average distance to the center is $(\mathrm{R})$, and the average distance of any two points is $(\mathrm{D})$, which happens to be the same as $D 3(A, B)$. Many implementation attempts (such as sklearn's) of BIRCH simply use the distance between the two cluster centers instead (E) - this cannot be defined in the original BIRCH architecture but is easy to add. 


$$
\begin{aligned}
\mathrm{R}(A B) & =\mathrm{R}(A, B)=\sqrt{\frac{1}{n_{A B}} \sum_{x \in A B}\left\|x-\mu_{A B}\right\|^{2}} \\
\mathrm{D}(A B) & =\mathrm{D} 3(A, B)=\sqrt{\frac{1}{n_{A B}\left(n_{A B}-1\right)} \sum_{x, y \in A B}\|x-y\|^{2}} \\
\mathrm{E}(A, B) & =\mathrm{D} 0(A, B)=\left\|\mu_{A}-\mu_{B}\right\|
\end{aligned}
$$

The values of $\mathrm{D}$ and $\mathrm{R}$ are almost identical (use Eq. 13 below): they differ only by a factor of $\frac{2 n}{n-1}$; similar to the regular radius and diameter. Because of the way they are used in BIRCH, we cannot expect them to perform very differently.

\subsection{Catastrophic Cancellation in BIRCH}

The numerical problem in BIRCH arises from the "textbook" equation for variance, $\operatorname{Var}(X)=E\left[X^{2}\right]-E[X]^{2}$. This equation-while mathematically correct - is prone to catastrophic cancellation when used with floating-point arithmetic, unless $E[X]^{2} \ll E\left[X^{2}\right]$ holds [20]. In clustering, we cannot assume that all clusters are close to the origin, and the ideal leaves have a small variance and represent the data by their differences in the mean. Because of this, it may not be sufficient to center the data globally. Furthermore, we do not know the center beforehand, and in BIRCH we only want to do a single pass over the data for performance.

Unfortunately, both of the original absorption criteria $\mathrm{R}$ and $\mathrm{D}$, as well as distance measures D2-D4 are computed using above "textbook" equality

$$
\operatorname{Var}(X)=\frac{1}{2 n^{2}} \sum_{x, y \in X}\|x-y\|^{2}=\frac{1}{n} \sum_{x \in X}\left\|x-\mu_{X}\right\|^{2}
$$

which yields the following equalities for BIRCH (equivalent to $n \cdot \operatorname{Var}(X)=S$ )

$$
S=\sum_{x \in X}\left\|x-\mu_{X}\right\|^{2}=\frac{1}{2 n} \sum_{x \in X}\|x-y\|^{2}=S S-\frac{1}{n}\|L S\|^{2} .
$$

The BIRCH authors hence proposed to compute these measures (we omit D0, D1, and E as they do not involve squares) based on clustering features as:

$$
\begin{aligned}
\mathrm{D} 2(A, B) & =\sqrt{\frac{1}{N_{A} N_{B}}\left(N_{B} S S_{A}+N_{A} S S_{B} \unlhd^{-} L S_{A}^{T} L S_{B}\right)} \\
\mathrm{D} 3(A, B) & =\sqrt{\frac{2}{N_{A}+N_{B}-1}\left(S S_{A}+S S_{B} \triangleq \frac{1}{N_{A}+N_{B}}\left\|L S_{A}+L S_{B}\right\|^{2}\right)} \\
\mathrm{D} 4(A, B) & =\sqrt{\frac{1}{N_{A}}\left\|L S_{A}\right\|^{2}+\frac{1}{N_{B}}\left\|L S_{B}\right\|^{2} \triangleq \frac{1}{N_{A}+N_{B}}\left\|L S_{A}+L S_{B}\right\|^{2}} \\
\mathrm{R}(A B) & =\sqrt{\frac{1}{N_{A B}}\left(S S_{A B} \triangleq \frac{1}{N_{A B}}\left\|L S_{A B}\right\|^{2}\right)} \\
\mathrm{D}(A B) & =\sqrt{\frac{2}{N_{A B}-1}\left(S S_{A B} \triangleq \frac{1}{N_{A B}}\left\|L S_{A B}\right\|^{2}\right)}
\end{aligned}
$$

The subtractions flagged with a warning symbol $₫$ can suffer from catastrophic cancellation and hence numerical problems. It may come unexpected that in the "variance increase" equation (D4) all SS terms cancel out, and we only get the vector product of the linear sums, but this is the Konig-Huygens theorem. 
The effect of the catastrophic cancellation usually leads to an underestimation of the actual variance, and hence of the distances. Because of this, data points may be assigned to the wrong branch or node. While the result will not be completely off, it is easy to avoid these problems in the first place. More severe problems arise when using the resulting variance in the subsequent steps, such as in clustering. Because most implementations of BIRCH only use the centers of the leaf entries for clustering (e.g., sklearn does not even use the weight, and only supports Euclidean distance D0), this has not been observed frequently.

Much of the later work based on BIRCH is prone to the same problem in one way or another. In CF-kernel density estimation [25], the variance is bounded to guarantee approximation quality - underestimating the variance invalidates this guarantee. The (diagonal) Mahalanobis distance used in [6] divides by the standard deviation, which can become 0 due to instabilities; the division tends to amplify the errors. CluStream [1] uses the standard deviation of the arrival times, estimated with the unstable equation. HPStream [2] relies on per attribute standard deviations for subspace clustering. DenStream [9] uses the radius $R$ to estimate density, while data bubbles [8] rely on the standard deviation to estimate the extent. ClusTree [17] estimates the variance in this unstable way. All of these methods can easily be modified to use BETULA cluster features.

Using the improved BETULA cluster features introduced in Section 3.3, which we will simply denote by $\mathrm{CF}$, we can easily avoid these numerical problems, because these features directly aggregate the squared errors instead of the sum of squares, as previously used for online estimation of variance [20].

\subsection{Improved Distance Computations}

In BETULA cluster features, we use the mean $\mu$ instead of the linear sum because this makes the subsequent operations more efficient (and elegant). The update equations for merging CFs also involve the mean (c.f. Eq. 4), and we can now compute the BIRCH distances in a more numerically stable way. Using BETULA cluster features $\mathrm{CF}=(n, \mu, S)$, and Eq. (14), we can compute the distances and absorption criteria now as follows (the derivation is included in the arXiv draft):

$$
\begin{aligned}
\operatorname{D} 0(A, B) & =\left\|\mu_{A}-\mu_{B}\right\| \\
\operatorname{D} 1(A, B) & =\left\|\mu_{A}-\mu_{B}\right\|_{1} \\
\operatorname{D} 2(A, B) & =\sqrt{\frac{1}{n_{A}} S_{A}+\frac{1}{n_{B}} S_{B}+\left\|\mu_{A}-\mu_{B}\right\|^{2}} \\
\operatorname{D} 3(A, B) & =\sqrt{\frac{2}{n_{A B}\left(n_{A B}-1\right)}\left(n_{A B}\left(S_{A}+S_{B}\right)+n_{A} n_{B}\left\|\mu_{A}-\mu_{B}\right\|^{2}\right)} \\
\operatorname{D} 4(A, B) & =\sqrt{\frac{n_{A} n_{B}}{n_{A B}}\left\|\mu_{A}-\mu_{B}\right\|^{2}} \\
\mathrm{R}_{A B} & =\sqrt{\frac{1}{n_{A B}} S_{A B}}=\sqrt{\frac{1}{n_{A B}}\left(S_{A}+S_{B}+\frac{n_{A} \cdot n_{B}}{n_{A B}}\left\|\mu_{A}-\mu_{B}\right\|^{2}\right)} \\
\mathrm{D}_{A B} & =\sqrt{\frac{2}{\left(n_{A B}-1\right)} S_{A B}}=\mathrm{D} 3(A, B)
\end{aligned}
$$

With these numerically more stable equations, we can build a CF-Tree using BETULA cluster features instead of the original BIRCH clustering features. 


\subsection{Gaussian Mixture Modeling with BETULA Cluster Features}

Gaussian Mixture Modeling (GMM) with the EM algorithm [11] is a popular, but fairly expensive clustering algorithm. Every iteration, the probability density functions (pdfs) of each Gaussian are evaluated at every data point, then the distribution parameters are updated based on all points weighted by their probabilities. Because this is a soft clustering, a tolerance threshold or an iteration limit are used for convergence. Formally, the method is linear in the number of data points, but in practice, it is fairly expensive because of the many pdfs to compute and the number of iterations. To scale this algorithm to large data sets (large $n$ ) as well as many clusters $k$, it is beneficial to use a data summarization technique such as BIRCH or BETULA. Several variations of GMM exist: we can restrict cluster shapes and we can have independent or shared model parameters. MAP estimation can be employed to improve the robustness [14], because there are other numerical pitfalls that can lead to degenerate clusters. We only consider some of the more popular variants in this work: the spherical model with varying weight and identical volume in each dimension (IGMM), the diagonal model with varying weight and different volume in each dimension (DGMM), and the fully variable model that models covariance (CGMM). If we only have a scalar for $S S$, then this is well-suited for the simplest model: A spherical model, in which the direction of variance does not matter. When using a vector for $S$, we can incorporate this per-axis information into the cluster models. For the arbitrarily oriented model, we would need to use a covariance in each cluster feature. This is possible using the corresponding equations for the covariance of [20], but the memory requirement increases to $1+d+\left(\begin{array}{c}d \\ 2\end{array}\right)=1+\frac{d(d+1)}{2}$ values per cluster feature. Because of this, we do not include this in the experiments.

For clustering, the main tree structure is usually discarded, and only the cluster features within the leaf nodes are kept. For the initialization of the algorithm, we apply the kmeans++ [4] initialization on the leaf entries. Afterward, the Gaussian Mixture Modeling algorithm is executed.

In classic GMM, we usually process a single data sample at a time. When processing cluster features, these represent multiple objects. To improve the quality of the clustering, rather than just using the cluster mean to represent a Cluster Feature, we use the Gaussian distribution of the data in the CF, which we assume is better (at least for GMM). To estimate the responsibilities of each cluster for each clustering we then use $\int_{x} \mathcal{N}\left(x \mid \mu_{1}, \sigma_{1}^{2}\right) \mathcal{N}\left(x \mid \mu_{2}, \sigma_{2}^{2}\right) d x=$ $\mathcal{N}\left(\mu_{1} \mid \mu_{2}, \sigma_{1}^{2}+\sigma_{2}^{2}\right)$. Using the law of total probability, these values are normalized to sum to 1, exactly as in the usual EM procedure. When updating the cluster models, the weight of the cluster features is trivially usable as additional weight, and we can update the model variance using Eq. (4).

By utilizing BETULA cluster features and EM-GMM it is possible to cluster big data sets with limited memory and high numerical stability as shown in Section 4. It is also possible to distribute this procedure into a cluster by partitioning the data and aggregating the models of all nodes (c.f. [20]). 


\section{Evaluation}

We compare the following alternative implementations of GMM:

Textbook Standard EM [11] using the equation $E\left[X^{2}\right]-E[X]^{2}$

Stable Numerically stable EM implementation (from ELKI $[21,20]$ )

$\mathrm{BIRCH}$ EM-style using the original BIRCH clustering features

BETULA EM-style using our new BETULA cluster features

The evaluation of clustering algorithms is inherently difficult because they are used in an unsupervised context, where no labeled data is available. Real data is usually dirty and contains undesirable artifacts (such as anomalies, duplicate values, and discretization effects) that can cause problems for methods that assume continuous data. GMM is no exception: e.g., constant attributes will break many implementations. In these experiments, we do not aim at showing the superiority of Gaussian Mixture Modeling over other approaches. The limitations of it are well understood, in particular when data has non-convex clusters.

Instead, we focus on the following research questions:

RQ1 How numerically (un-)stable is BIRCH, does BETULA help?

RQ2 Is the quality of BETULA comparable with BIRCH and regular GMM?

RQ3 How does BETULA scale with data set size (and compare to BIRCH)?

RQ4 Are the results applicable to real data?

\subsection{Experimental Setup}

We modify the existing implementations of BIRCH and GMM clustering of ELKI 0.7.5 [21]. By keeping most of the code shared, we try to minimize the effects caused by implementation differences, as recommended for comparing algorithms [18]. All computations are executed on a small cluster with Intel E5-2697v2 CPUs, we do not use multithreading, and we repeated each experiment 10 times with varying random seeds and data input order, and give the average results. All the CF-Trees are built using the variance-increase distance (D4, Eq. 9) in combination with the radius absorption criterion (R, Eq. 10). This combination yields subclusters with low variance as input for the GMM clustering. We do not present results with other distances and absorption criteria here because of redundancy; they were similar. The size of CF-Trees is by default limited to 5000 leaf entries unless specified differently; when this number is exceeded the tree is rebuilt with a bigger threshold as in BIRCH. For the GMM clustering step, all algorithms are initialized by kmeans++ [4]. After 100 iterations or when no further improvement can be made the optimization is stopped.

\subsection{Numerical Stability}

First, we demonstrate the numerical instability using synthetic data with two Gaussian clusters in $\mathbb{R}^{3}$ of 150000 points. Both clusters have standard deviations $\left[\frac{4}{3}, 1, \frac{3}{4}\right]$, and the only variable in the test is how far the clusters are shifted away from the mean. For small separation, both clusters overlap but with increasing distance, the clustering gets trivial until numerical stability comes into play. 
The impact of the increasing distance between the clusters can be seen in Fig. 1 where all algorithms provide good results until first the Textbook IGMM implementation at $5 \cdot 10^{6}$ and then BIRCH IGMM at $2 \cdot 10^{7}$ begin to deteriorate. The degeneration of BIRCH IGMM begins a bit later than Textbook IGMM because of the aggregation in the CF-Tree helping a bit, but it then fails even worse. A deterioration at $10^{7}$ is to be expected from double-precision because of the squared values; with single-precision floating-point, it is to be expected to occur at a separation of $10^{3}$. Both the "Stable" regular GMM and BETULA are not affected and solve this idealized toy problem without difficulties (RQ1).

\subsection{Quality Comparison on Synthetic Data}

We now address the question of result quality (RQ2) in a scenario where all algorithms are stable. For the evaluation, two synthetic data sets are used, which are similar to data used for the evaluation of the original BIRCH algorithm [23] but larger and with increased variability. We use the data generator of ELKI [21], which has a convenient size multiplier parameter for this experiment.

The first data set is called "Grid" and consists of a 10 by 10 grid of clusters with a distance of 5 between the means of the clusters on each axis. Each cluster consists of 10000 points with a variance per attribute randomly drawn from $\mathcal{N}(1,0.25)$. The second data set, "Random", consists of 100 clusters in a 50 by 50 area with the cluster means distributed by Halton sampling, which produces a pseudo-random uniform distribution. The variance of each cluster is again specified by a normal distribution $\mathcal{N}(1,0.15)$. This time the size of each cluster varies and is randomly drawn from between 5000 and 15000 points.

Fig. 2 shows the log-likelihood of the models on these data sets. For both, it can be seen that the data set size has next to no influence on the quality of the fit. The models with diagonal variance (Stable DGMM and BETULA DGMM) produce a better fit than the models that are restricted to using the same variance in each attribute. On the "Random" data set, all IGMM approaches perform similar (as expected). On the "Grid" data set, both BETULA IGMM and BIRCH IGMM unexpectedly achieve a higher likelihood than the standard IGMM algorithms. This difference can be explained by the fact that the implementations using cluster features converge faster (because there are fewer objects) than the approaches that use the raw data; the latter have not yet converged within the

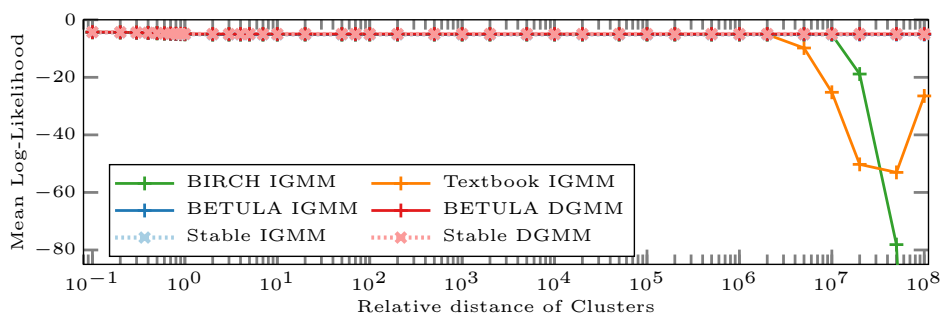

Fig. 1. The log-likelihood goodness of fit of the model with increasing distance between the clusters demonstrates the numerical instability of some algorithms. 


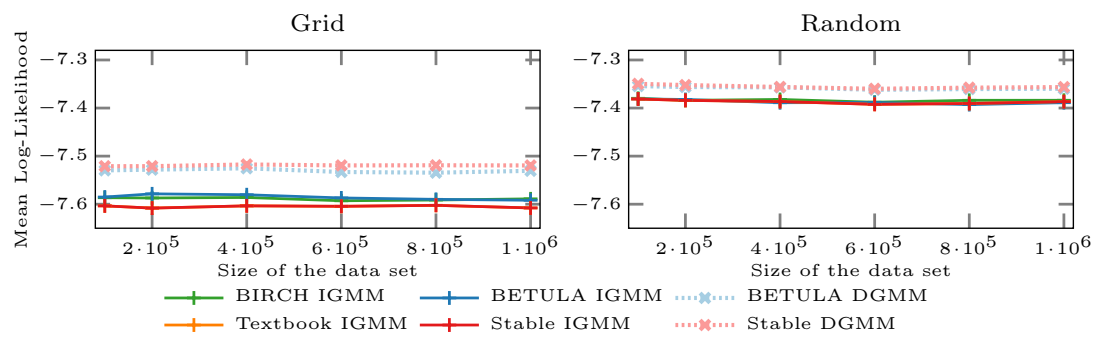

Fig. 2. Log-Likelihood goodness of fit of the model on both synthetic data sets.

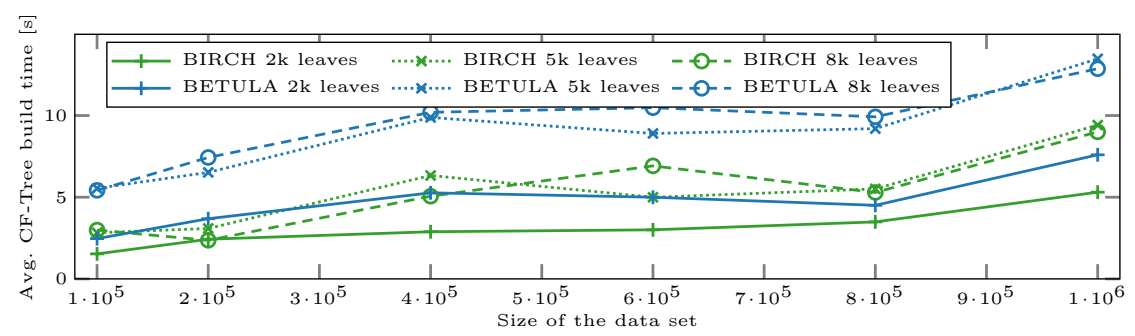

Fig. 3. Build time with a varying number of leaf entries on the random data set.

maximum number of iterations. However, this experiment is designed to test if BETULA performs similar to BIRCH on the same test data that the BIRCH publications used, and to detect programming errors.

\subsection{Runtime Evaluation on Synthetic Data}

When evaluating the runtime of BETULA with GMM clustering two measurements are of interest: The time to build the CF-Tree only, and the time for the entire clustering procedure. Fig. 3 shows the time BETULA and BIRCH need to build the CF-Tree for various tree sizes. It can be seen that the time for building the tree increases with the size of the data set and also with the size of the tree due to an increasing number of distance calculations for the insertion of new points. The construction time for BETULA is larger than for BIRCH because this implementation uses a vector for storing the variances, while the BIRCH implementation uses only a scalar; but the tree construction is only a small part of the total time as we will see next.

When looking at the complete runtime of BIRCH (respectively BETULA) including GMM clustering, shown in Fig. 4, we can see that the standard GMM algorithms have a much higher runtime by a factor of 18 to 52 on this data set, due to the compression achieved by the CF-Tree (which improves with data set size). We use a log-log plot to see the differences between BIRCH and BETULA, which perform very similar (RQ3). BETULA is up to $5 \%$ faster than BIRCHdespite using a vector to store variances - because the BETULA cluster features can be used directly for clustering, while more additional computations are necessary with BIRCH clustering features to obtain mean and variance on the fly. 


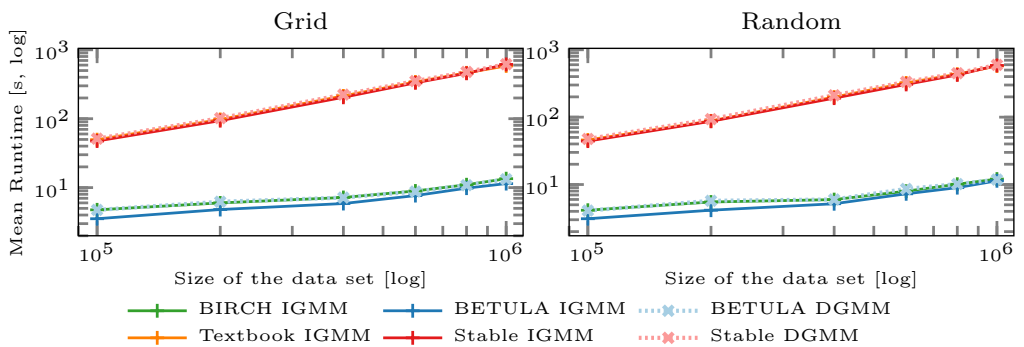

Fig. 4. Runtime of the clustering on both synthetic data sets.

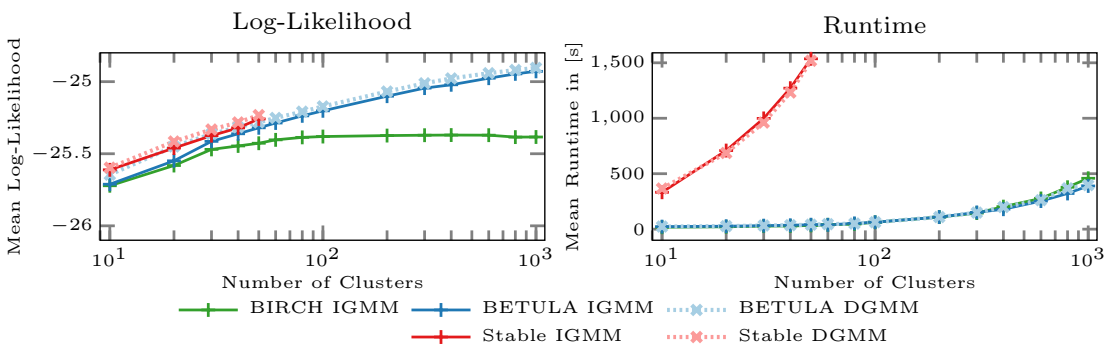

Fig. 5. Log-Likelihood goodness of fit of the models and runtime on the traffic accident data with 15000 leaf entries (Stable GMM only up to 50 clusters because of runtime).

\subsection{Clustering Real Data}

To test the algorithm on real data, we use the location information of the UK "Road Safety Data" from 1979 to 2004 from data.gov.uk. ${ }^{1}$ This data set has about 6.2 million entries and contains data on road accidents from Great Britain. The location information in this data set is given in the OSGR grid reference system which is only used in Great Britain; which we convert to the appropriate UTM coordinate system. For this experiment, we reduced the cluster feature precision from double precision to single precision in both BIRCH and BETULA to demonstrate the numerical instabilities on real data. The regular GMM clustering is performed with double precision to get a more precise reference value.

Fig. 5 shows that Stable DGMM and Stable IGMM achieve a better fit to the data than the CF-Tree based approximations (which is to be expected, given that they use the individual points and double precision). However, the runtime of this method is much higher, and hence it is only computed up to $k=50$ clusters. BETULA with DGMM and IGMM clustering obtain only slightly worse results, showing that the BETULA cluster features provide a reasonably close approximation of the data. BIRCH IGMM on the other hand shows its numerical instabilities and with an increasing number of clusters, the quality deteriorates compared to BETULA. For numerous clusters (and a large value makes sense on this data set), BETULA with DGMM delivers the best results at an acceptable run time: As seen in Fig. 5, all GMM with Stable, BIRCH, and

\footnotetext{
${ }^{1}$ https://data.gov.uk/dataset/cb7ae6f0-4be6-4935-9277-47e5ce24a11f
} 


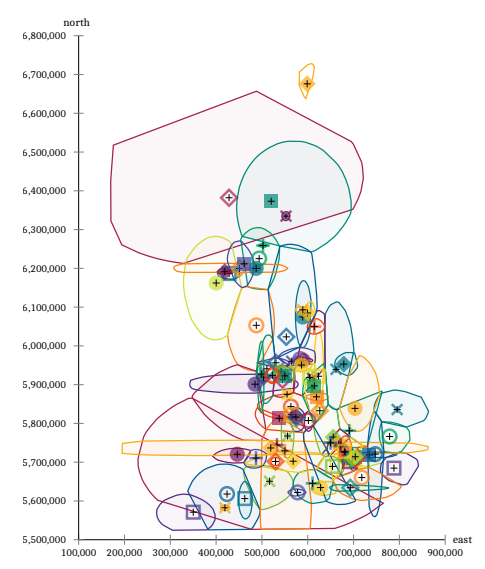

Fig. 6. Convex hulls of clusters with BETULA DGMM on the traffic accident data with 100 clusters (three clusters omitted for a cleaner visualization).

BETULA scale approximately linear in the number of clusters $k$; but because the CF-Trees reduce the data set from 6.2 million to at most 15000 cluster features (a factor of over 400), we obtain good results at a much smaller run time than with regular Stable DGMM or IGMM (RQ4).

Fig. 6 shows the convex hulls and cluster centroids of an exemplary clustering of the traffic data set with $k=100$ clusters, using BETULA DGMM and visualized with ELKI. We removed three clusters containing only input data errors to improve readability. The shape of Great Britain can be recognized; small and dense clusters are found around the larger British cities, especially London. Larger clusters with lower density on the other hand cover rural areas with fewer accidents (it is typical behavior of GMM to nest dense clusters with low variance inside "background" clusters with high variance and fewer data points).

\section{Conclusion}

Big data analysis and data stream clustering are hot topics in today's research. The CF-Tree of BIRCH is a popular technique for this that inspired many subsequent works. Recently, the reliability of machine learning is receiving increased attention; unfortunately, we found that "catastrophic cancellation" is a major problem when calculating variances in BIRCH and derived methods, which can cause the results to deteriorate.

In this article, we proposed BETULA cluster features, that can serve as a drop-in replacement for BIRCH. These do no longer exhibit this problem as they avoid using the unstable equation, at a negligible performance difference. We also show how to use BETULA to accelerate Gaussian Mixture Modeling, while using the variance information from the cluster features for improved quality, compared to the standard approach of only using the centroids of each leaf entry. 


\section{References}

1. Aggarwal, C.C., Han, J., Wang, J., Yu, P.S.: A framework for clustering evolving data streams. In: VLDB (2003)

2. Aggarwal, C.C., Han, J., Wang, J., Yu, P.S.: A framework for projected clustering of high dimensional data streams. In: VLDB (2004)

3. Ankerst, M., Breunig, M.M., Kriegel, H., Sander, J.: OPTICS: ordering points to identify the clustering structure. In: SIGMOD (1999)

4. Arthur, D., Vassilvitskii, S.: k-means++: the advantages of careful seeding. In: SODA (2007)

5. Bonner, R.E.: On some clustering techniques. IBM Journal of Research and Development 8(1) (1964)

6. Bradley, P.S., Fayyad, U.M., Reina, C.: Scaling clustering algorithms to large databases. In: KDD. pp. 9-15 (1998)

7. Breunig, M.M., Kriegel, H., Kröger, P., Sander, J.: Data bubbles: Quality preserving performance boosting for hierarchical clustering. In: SIGMOD (2001)

8. Breunig, M.M., Kriegel, H., Sander, J.: Fast hierarchical clustering based on compressed data and OPTICS. In: PKDD (2000)

9. Cao, F., Ester, M., Qian, W., Zhou, A.: Density-based clustering over an evolving data stream with noise. In: SDM (2006)

10. Chiu, T., Fang, D., Chen, J., Wang, Y., Jeris, C.: A robust and scalable clustering algorithm for mixed type attributes in large database environment. In: KDD (2001)

11. Dempster, A.P., Laird, N.M., Rubin, D.B.: Maximum likelihood from incomplete data via the EM algorithm. J. Royal Stat. Soc., Series B 39(1) (1977)

12. Estivill-Castro, V.: Why so many clustering algorithms: a position paper. SIGKDD Explorations 4(1) (2002)

13. Fichtenberger, H., Gillé, M., Schmidt, M., Schwiegelshohn, C., Sohler, C.: BICO: BIRCH meets coresets for k-means clustering. In: ESA (2013)

14. Fraley, C., Raftery, A.E.: Bayesian regularization for normal mixture estimation and model-based clustering. J. Classification 24(2) (2007)

15. Ganti, V., Gehrke, J., Ramakrishnan, R.: DEMON: mining and monitoring evolving data. IEEE Trans. Knowl. Data Eng. 13(1) (2001)

16. Han, J., Kamber, M., Pei, J.: Data Mining: Concepts and Techniques, 3rd edition. Morgan Kaufmann (2011)

17. Kranen, P., Assent, I., Baldauf, C., Seidl, T.: The ClusTree: indexing micro-clusters for anytime stream mining. Knowl. Inf. Syst. 29(2) (2011)

18. Kriegel, H., Schubert, E., Zimek, A.: The (black) art of runtime evaluation: Are we comparing algorithms or implementations? Knowl. Inf. Syst. 52(2) (2017)

19. Ng, R.T., Han, J.: CLARANS: A method for clustering objects for spatial data mining. IEEE Trans. Knowl. Data Eng. 14(5) (2002)

20. Schubert, E., Gertz, M.: Numerically stable parallel computation of (co-)variance. In: SSDBM (2018)

21. Schubert, E., Zimek, A.: ELKI: A large open-source library for data analysis ELKI release 0.7.5 "Heidelberg". CoRR abs/1902.03616 (2019)

22. Zhang, T.: Data clustering for very large datasets plus applications. Tech. Rep. 1355, University of Wisconsin Madison (1996)

23. Zhang, T., Ramakrishnan, R., Livny, M.: BIRCH: an efficient data clustering method for very large databases. In: SIGMOD (1996)

24. Zhang, T., Ramakrishnan, R., Livny, M.: BIRCH: A new data clustering algorithm and its applications. Data Min. Knowl. Discov. 1(2) (1997)

25. Zhang, T., Ramakrishnan, R., Livny, M.: Fast density estimation using CF-kernel for very large databases. In: KDD (1999) 


\section{A Derivation of the BETULA Distance Criteria}

The derivations rely on the following two equivalences:

$$
\begin{array}{r}
\sum_{i=1}^{n} \sum_{j=1}^{n}\left\|x_{i}-x_{j}\right\|^{2}=2 n \sum_{i=1}^{n}\left\|x_{i}-\mu\right\|^{2} \\
S_{A B}=S_{A}+S_{B}+\frac{n_{A} n_{B}}{n_{A}+n_{B}}\left\|\mu_{A}-\mu_{B}\right\|^{2}
\end{array}
$$

We can now derive the following equations to compute the distances using BETULA cluster features. First, average inter-cluster distance:

$$
\begin{aligned}
\mathrm{D} 2(A, B)^{2} & =\frac{1}{n_{A} n_{B}} \sum_{x \in A} \sum_{y \in B}\|x-y\|^{2} \\
& =\frac{1}{2 n_{A} n_{B}}\left(\sum_{x, y \in A B}\|x-y\|^{2}-\sum_{x, y \in A}\|x-y\|^{2}-\sum_{x, y \in B}\|x-y\|^{2}\right) \\
& =\frac{1}{n_{A} n_{B}}\left(n_{A B} \sum_{x \in A B}\|x-\mu\|^{2}-n_{A} \sum_{x \in A}\|x-\mu\|^{2}-n_{B} \sum_{x \in B}\|x-\mu\|^{2}\right) \\
& =\frac{1}{n_{A} n_{B}}\left(n_{A B} S_{A B}-n_{A} S_{A}-S_{B}\right) \\
& =\frac{1}{n_{A} n_{B}}\left(n_{A B}\left(S_{A}+S_{B}+\frac{n_{A} n_{B}}{n_{A}+n_{B}}\left\|\mu_{A}-\mu_{B}\right\|^{2}\right)-n_{A} S_{A}-S_{B}\right) \\
& =\frac{n_{B}}{n_{A} n_{B}} S_{A}+\frac{n_{A}}{n_{A} n_{B}} S_{B}+\frac{n_{A} n_{B}}{n_{A} n_{B}}\left\|\mu_{A}-\mu_{B}\right\|^{2} \\
& =\frac{1}{n_{A}} S_{A}+\frac{1}{n_{B}} S_{B}+\left\|\mu_{A}-\mu_{B}\right\|^{2}
\end{aligned}
$$

Second, average intra-cluster distance:

$$
\begin{aligned}
\operatorname{D} 3(A, B)^{2} & =\frac{1}{n_{A B}\left(n_{A B}-1\right)} \sum_{x, y \in A B}\|x-y\|^{2}=\frac{2}{n_{A B}\left(n_{A B}-1\right)} n_{A B} \sum_{x \in A B}\|x-\mu\|^{2} \\
& =\frac{2}{n_{A B}\left(n_{A B}-1\right)} n_{A B} S_{A B} \\
& =\frac{2}{n_{A B}\left(n_{A B}-1\right)} n_{A B}\left(S_{A}+S_{B}+\frac{n_{A} n_{B}}{n_{A B}}\left\|\mu_{A}-\mu_{B}\right\|^{2}\right) \\
& =\frac{2}{n_{A B}\left(n_{A B}-1\right)}\left(n_{A B}\left(S_{A}+S_{B}\right)+n_{A} n_{B}\left\|\mu_{A}-\mu_{B}\right\|^{2}\right)
\end{aligned}
$$

Third, variance-increase distance:

$$
\begin{aligned}
\mathrm{D} 4(A, B)^{2} & =\sum_{x \in A B}\left\|x-\mu_{A B}\right\|^{2}-\sum_{x \in A}\left\|x-\mu_{A}\right\|^{2}-\sum_{x \in B}\left\|x-\mu_{B}\right\|^{2} \\
& =S_{A B}-S_{A}-S_{B}=S_{A}+S_{B}+\frac{n_{A} n_{B}}{n_{A B}}\left\|\mu_{A}-\mu_{B}\right\|^{2}-S_{A}-S_{B} \\
& =\frac{n_{A} n_{B}}{n_{A B}}\left\|\mu_{A}-\mu_{B}\right\|^{2}
\end{aligned}
$$

Last, radius (average distance to the center):

$$
\begin{aligned}
\mathrm{R}(A, B)^{2} & =\frac{1}{n_{A B}} \sum_{x \in A B}\left\|x-\mu_{A B}\right\|^{2}=\frac{1}{n_{A B}} S_{A B} \\
& =\frac{1}{n_{A B}}\left(S_{A}+S_{B}+\frac{n_{A} \cdot n_{B}}{n_{A B}}\left\|\mu_{A}-\mu_{B}\right\|^{2}\right)
\end{aligned}
$$

We do not provide derivations for Euclidean distance D0, Manhattan distance $D 1$, as these can trivially be computed from the cluster means $\mu$ using the standard definitions of these distances. The diameter $D$ is equivalent to $D 3$. 


\section{B Derivation of the BIRCH Distance Criteria}

The derivations rely on the following equivalence:

$$
\frac{1}{n} \sum_{i=1}^{n}\|x-\mu\|^{2}=\operatorname{Var}\left(X^{2}\right)=E\left(X^{2}\right)-E(X)^{2}=\frac{1}{n} S S-\left\|\frac{L S}{n}\right\|^{2}
$$

Using this - numerically problematic - equivalence we can reproduce the equations used in the original BIRCH algorithm [22]:

$$
\begin{aligned}
\mathrm{D} 2(A, B)^{2} & =\frac{1}{n_{A} n_{B}} \sum_{x \in A} \sum_{y \in B}\|x-y\|^{2} \\
& =\frac{1}{2 n_{A} n_{B}}\left(\sum_{x, y \in A B}\|x-y\|^{2}-\sum_{x, y \in A}\|x-y\|^{2}-\sum_{x, y \in B}\|x-y\|^{2}\right) \\
& =\frac{1}{n_{A} n_{B}}\left(n_{A B} \sum_{x \in A B}\|x-\mu\|^{2}-n_{A} \sum_{x \in A}\|x-\mu\|^{2}-n_{B} \sum_{x \in B}\|x-\mu\|^{2}\right) \\
& =\frac{1}{n_{A} n_{B}}\left(\left(n_{A B} S S_{A B}-\left\|L S_{A B}\right\|^{2}\right)-\left(n_{A} S S_{A}-\left\|L S_{A}\right\|^{2}\right)-\left(n_{B} S S_{B}-\left\|L S_{B}\right\|^{2}\right)\right) \\
& =\frac{1}{n_{A} n_{B}}\left(n_{B} S S_{A}+n_{A} S S_{B}+\left(\left\|L S_{A}\right\|^{2}+\left\|L S_{B}\right\|^{2}-\left\|L S_{A}+L S_{B}\right\|^{2}\right)\right) \\
& =\frac{1}{n_{A} n_{B}}\left(n_{B} S S_{A}+n_{A} S S_{B}-2 L S_{A}^{T} L S_{B}\right) \\
\mathrm{D} 3(A, B)^{2} & =\frac{1}{n_{A B}\left(n_{A B}-1\right)} \sum_{x, y \in A B}\|x-y\|^{2}=\frac{2 n_{A B}}{n_{A B}\left(n_{A B}-1\right)} \sum_{x \in A B}\|x-\mu\|^{2} \\
& =\frac{2}{n_{A B}-1}\left(S S_{A B}-n_{A B}\left\|\frac{L S_{A B}}{n_{A B}}\right\|^{2}\right) \\
& =\frac{2}{n_{A}+n_{B}-1}\left(S S_{A}+S S_{B}-\frac{1}{n_{A}+n_{B}}\left\|L S_{A}+L S_{B}\right\|^{2}\right) \\
\mathrm{D} 4(A, B)^{2} & =\sum_{x \in A B}\left\|x-\mu_{A B}\right\|^{2}-\sum_{x \in A}\left\|x-\mu_{A}\right\|^{2}-\sum_{x \in B}\left\|x-\mu_{B}\right\|^{2} \\
& =S S_{A B}-\frac{1}{n_{A B}}\left\|L S_{A B}\right\|^{2}-\left(S S_{A}-\frac{1}{n_{A}}\left\|L S_{A}\right\|^{2}\right)-\left(S S_{B}-\frac{1}{n_{B}}\left\|L S_{B}\right\|^{2}\right) \\
& =S S_{A B}-S S_{A}-S S_{B}+\frac{1}{n_{A}}\left\|L S_{A}\right\|^{2}+\frac{1}{n_{B}}\left\|L S_{B}\right\|^{2}-\frac{1}{n_{A B}}\left\|L S_{A B}\right\|^{2} \\
& =\frac{1}{n_{A}}\left\|L S_{A}\right\|^{2}+\frac{1}{n_{B}}\left\|L S_{B}\right\|^{2}-\frac{1}{n_{A}+n_{B}}\left\|L S_{A}+L S_{B}\right\|^{2} \\
\mathrm{R}(A, B)^{2} & =\frac{1}{n_{A B}} \sum_{x \in A B}\left\|x-\mu_{A B}\right\|^{2}=\frac{1}{n_{A B}} S S_{A B}-\left\|\frac{L S_{A B}}{n_{A B}}\right\|^{2} \\
& =\frac{1}{n_{A B}}\left(S S_{A B}-\frac{1}{n_{A B}}\left\|L S_{A B}\right\|^{2}\right)
\end{aligned}
$$

